\title{
Lifetime Self-Harm Behaviors Are Not More Prevalent in Bariatric Surgery Candidates than in Community Controls with Obesity
}

\author{
Astrid Müller ${ }^{a}$ Laurence Claes $^{b, c}$ Dirk Smits ${ }^{b, d}$ Kathrin Schag ${ }^{e}$ \\ Martina de Zwaan ${ }^{a}$ \\ a Department of Psychosomatic Medicine and Psychotherapy, Hannover Medical School, \\ Hanover, Germany; ${ }^{b}$ Faculty of Psychology and Educational Sciences, University of Leuven, \\ Leuven, Belgium; ' ${ }^{C}$ Faculty of Medicine and Health Sciences, University Antwerp, Antwerp, \\ Belgium; ${ }^{d}$ Odisee University College, Brussels, Belgium; e Department of Psychosomatic \\ Medicine and Psychotherapy, University Hospital Tübingen, Tübingen, Germany
}

\section{Keywords}

Obesity · Bariatric surgery $\cdot$ Self-harm $\cdot$ Suicide attempts

\begin{abstract}
Objective: The study aimed at investigating the lifetime prevalence of 22 self-harm behaviors in bariatric surgery candidates (pre-bariatric surgery group; PSG) compared to community controls with obesity (obese community group; OCG). Methods: The Self-Harm Inventory (SHI) was administered to the PSG $\left(n=139, \mathrm{BMI} \geq 35 \mathrm{~kg} / \mathrm{m}^{2}\right)$ and to the OCG $(\mathrm{n}=122, \mathrm{BMI} \geq$ $35 \mathrm{~kg} / \mathrm{m}^{2}$ ). Results: Group comparison of cumulative SHI scores indicated a trend towards less endorsed SHI items in the PSG compared to the OCG (median $_{P S G}=1.00, I_{\text {Q }} R_{P S G}=2.00$, median $_{O C G}=1.00, I_{O R G}=2.25, U=7.241, p=0.033, \eta^{2}=0.02$ ). No significant group differences were found with regard to the rate of suicide attempts (12.4\% vs. 9.4\% for OCG vs. PSG). At least one type of lifetime self-harm behavior was admitted by $51.8 \%$ of the PSG and $63.9 \%$ of the OCG $\left(\chi^{2}(1)=3.91, p=0.048\right)$. The results of logistic regressions using Firth's bias reduction method with at least one $\mathrm{SHI}$ item endorsed as dependent variable, group as categorical predictor (PSG as baseline), and age or BMI or PHQ-4 as continuous control variable indicated that only PHQ-4 had a positive effect on the odds ratio. Conclusion: The results suggest that self-harm (including suicidal attempts) is not more prevalent in bariatric surgery candidates than in community control participants with obesity. Further studies are needed to investigate self-harm in bariatric surgery patients, prior and following surgery, compared to non-operated patients with obesity.

(C) 2018 The Author(s)

Published by S. Karger $\mathrm{GmbH}$, Freiburg
\end{abstract}


Müller et al.: Lifetime Self-Harm Behaviors Are Not More Prevalent in Bariatric Surgery Candidates than in Community Controls with Obesity

\section{Introduction}

Self-harm includes a broad range of direct and indirect self-damaging behaviors, regardless of their suicidal intent [1]. Direct self-harm refers to socially unaccepted, deliberate destruction of one's own body tissue such as cutting, burning, scratching, or biting [2]. Indirect self-harm includes self-damaging behaviors such as engagement in risky and reckless behaviors or abusive relationships, disordered eating, substance abuse etc. [2].

A growing body of literature indicates alarmingly high rates of emergency visits and inpatient contacts due to deliberate self-harm, including suicide attempts, following bariatric surgery [3-11]. Data sources for most of these studies were national patient registers covering psychiatric and/or somatic inpatient contacts and incidences of emergency visits due to selfharm and suicide attempts. There is no doubt that these data are very useful to estimate the course of self-harm and suicide attempts prior to and following bariatric surgery. Though, the data were not collected for research purposes, and the outcome 'emergency visits' may have mirrored only a certain proportion of self-harming events resulting in an underestimation of actual self-harm.

Studies using detailed assessment of self-harm among bariatric surgery patients are still scarce. Sansone et al. [12] investigated a broad range of 22 lifetime self-harming behaviors in a group of 121 bariatric surgery candidates ( 104 women, $17 \mathrm{men}$ ) with an average age of 44.6 years and a BMI ranging from 27.2 to $92.1 \mathrm{~kg} / \mathrm{m}^{2}$ by means of the Self-Harm Inventory (SHI) [1]. Almost half of the sample admitted at least one form of self-harm (46.3\%), most frequently sexual promiscuity $(22.3 \%)$, torturing oneself with self-defeating thoughts $(20.7 \%)$, alcohol abuse (19.0\%), and engaging in emotionally abusive relationships (16.5\%). In terms of suicidality during the lifespan, $9.1 \%$ of the patients admitted suicide attempts, and $9.1 \%$ acknowledged histories of overdoses [12]. Sansone et al. [12] assumed that the prevalence rate of suicide attempts in this pre-bariatric surgery group exceeded the rate in the general population [12]. However, the study was limited by the lack of a community-based comparison group.

The SHI was also used in a recent large-scale German population-based study $(\mathrm{N}=2,507$; age 14-94 years; $55.5 \%$ women) [13]. In this study, the following rates of at least one selfharm behavior during the lifespan were assessed within BMI groups: underweight $40 \%$, normal weight $50 \%$, overweight $46.3 \%$, class 1 obesity $52.9 \%$, class 2 obesity $60.8 \%$, and class 3 obesity $57.8 \%\left(\chi^{2}=12.96, d f=5, p=0.024\right)$ [13]. Between-group comparisons showed that individuals with class 2 obesity admitted more often any lifetime self-harm behavior than persons with underweight, normal weight, or overweight. With regard to continuous SHI total scores, it appeared that the class 2 obesity group reported higher SHI scores than all groups with a lower BMI, and that the class 3 obesity group had higher SHI scores than the overweight group [13].

The question arises, if patients seeking bariatric surgery suffer from equally or higher rates of self-harm than community controls with obesity. The present study aimed at addressing this question by comparing the lifetime prevalence of self-harm behaviors in bariatric surgery candidates and community control participants with obesity. In addition, current general psychopathology was assessed in both groups in order to adjust the analyses for this potential confounder. Based on previous studies reporting high psychiatric comorbidity [14-17] and elevated rates of self-harm [5,12,18], including suicide attempts [6], in patients undergoing bariatric surgery, it was hypothesized that preoperative patients will admit more self-harm behaviors during the lifespan than control persons with obesity and that higher frequencies of self-harm behaviors will be related to higher BMI in both groups. 
Müller et al.: Lifetime Self-Harm Behaviors Are Not More Prevalent in Bariatric Surgery Candidates than in Community Controls with Obesity

\section{Participants and Methods}

\section{Participants}

The pre-bariatric surgery group (PSG) was recruited within the routine preoperative psychiatric evaluation at Hannover Medical School between January and October 2015. Participation in the study was completely voluntary. The assessment was conducted by independent assessors who were not involved in the preoperative psychosomatic evaluation or in any kind of clinical routine. 139 patients (59\% of potential candidates for the study) aged between 18 and 63 years (77.7\% women) gave written informed consent for participation in the study according to procedures approved by the institutional ethics committee of the Hannover Medical School. All patients had a BMI of 35+ kg/m² (range 35.10-69.30 kg/m²). The majority $(92.1 \%)$ suffered from class 3 obesity (BMI $\geq 40 \mathrm{~kg} / \mathrm{m}^{2}$ ), and only $7.9 \%$ from class 2 obesity $\left(35 \mathrm{~kg} / \mathrm{m}^{2} \leq \mathrm{BMI}\right.$ $\leq 39.99 \mathrm{~kg} / \mathrm{m}^{2}$ ).

Data from the community sample were collected between March and May 2015 with the assistance of a demographic consulting company (USUMA, Berlin, Germany). The sampling procedure is described in detail elsewhere [13]. It included three consecutive steps: in the first step, a grid of 258 regional sampling areas was randomly selected from a roster of such non-overlapping grids that have been centrally assembled to enhance representativeness in stratified regional sampling in Germany. In the second step, a random procedure to select households of the respective area was implemented within all sampling areas. In the final step, one member of the selected household fulfilling the inclusion criteria (age 14 or older, able to read and understand the German language) was sampled randomly in a pre-specified standardized manner. A first attempt was made for 4,844 addresses. If not at home, a maximum of three attempts was made to contact the selected person. All subjects were visited by a study assistant who informed them about the investigation, obtained written informed consent, and presented them with the questionnaire. A total sample of 2,507 participants provided valid information. For the current study, only data from participants within the same age range as the preoperative group (i.e., 18-63 years) and with BMI $35+\mathrm{kg} / \mathrm{m}^{2}$ were selected, leaving a final obese community group (OCG) of 122 individuals (70.5\% women) aged between 18 and 63 years. BMI in this group ranged from 35.00 to $67.00 \mathrm{~kg} / \mathrm{m}^{2}, 69.7 \%$ of participants had class 2 obesity, and $30.3 \%$ had class 3 obesity.

\section{Measures}

Sociodemographic data, weight, and height were self-reported. The validated German translation [13] of the SHI [1] was used to measure self-harm. The SHI starts with the instruction: 'Please answer the following questions by checking either "Yes" or "No". Check "Yes" only to those items that you have done intentionally, or on purpose, to hurt yourself.' Each item is preceded by the phrase: 'Have you ever intentionally, or on purpose, ...'. The SHI contains of 22 items that ask for lifetime history of engagement in self-harm behaviors such as cutting oneself, scratching oneself, preventing wounds from healing, head banging, abusing alcohol, engaging in emotionally/sexually abusive relationships etc. The questionnaire also includes one item assessing lifetime suicide attempts (Item \#18: 'Have you ever intentionally or on purpose attempted suicide?'). The total SHI score is determined by the number of endorsed items and may range from 0 to 22 . Internal consistency coefficient for the SHI total score was $\alpha=0.80$ in the PSG, and $\alpha=0.88$ in the OCG.

The 4-item Patient Health Questionnaire for Depression and Anxiety (PHQ-4) [19] was used to assess general psychopathology over the last 2 weeks. This ultra-short screening instrument consists of two core general anxiety disorder items and two core depression items assessing symptom frequencies. Responses are scored from 0 ('not at all') to 3 ('nearly every day'). The total PHQ-4 score ranges from 0 to 12 ( $\alpha_{\mathrm{PSG}}=$ $\left.0.85, \alpha_{O C G}=0.84\right)$.

\section{Data Analysis}

Statistical analyses were conducted using IBM ${ }^{\circledR}$ SPSS $^{\circledR}$ Statistics Version 24.0 (IBM, Armonk, NY, USA). Age, BMI, PHQ-4, and SHI scores were not normally distributed (i.e., significant Kolmogorov-Smirnov test and significant Shapiro-Wilk test). The following descriptive statistics were used to summarize sociodemographic variables and questionnaire results: frequency and percentage for categorical variables, and median and interquartile range $(I Q R)$ for continuous variables. Group differences (PSG vs. OCG) were examined with $\chi^{2}$ test for categorical variables and Mann-Whitney U Test for continuous variables. $\eta^{2}$ was used as effect size (small effect $\eta^{2}=0.01$, medium effect $\eta^{2}=0.06$, large effect $\eta^{2}=0.14$ ) [20]. Group differences in prevalence rates of any lifetime self-harm were examined using binary logistic regressions using Firth's bias reduction method [21] with at least one SHI item endorsed as dependent variable, PSG versus OCG as categorical 
predictor, and age, BMI and PHQ-4 as continuous control variables. Relationships between variables were determined by calculating two-tailed Spearman's rank-order correlations using the list-wise deletion of missing data option. The significance level for $\alpha$ was set at $\mathrm{p}<0.01$ in order to correct for multiple comparisons.

\section{Results}

On a bivariate level, in both groups SHI total scores were not correlated with age (PSG: $\mathrm{r}_{\mathrm{S}}=-0.14, \mathrm{p}=0.093$; OCG: $\left.\mathrm{r}_{\mathrm{S}}=-0.02, \mathrm{p}=0.082\right)$ or BMI (PSG: $\mathrm{r}_{\mathrm{S}}=0.16, \mathrm{p}=0.064 ;$ OCG: $\mathrm{r}_{\mathrm{S}}=$ $-0.05, \mathrm{p}=0.571$ ), but with PHQ-4 scores (PSG: $\mathrm{r}_{\mathrm{S}}=0.47, \mathrm{p}<0.001$; OCG: $\mathrm{r}_{\mathrm{S}}=0.51, \mathrm{p}<0.001$ ). The groups did not differ with respect to gender, but the PSG was on average younger (median $_{\mathrm{PSG}}=40, \mathrm{IQR}_{\mathrm{PSG}}=16$, median $_{\mathrm{OCG}}=44, \mathrm{IQR}_{\mathrm{OCG}}=22, \mathrm{U}=6,582, \mathrm{p}=0.002$ ), included more individuals with class 3 obesity $\left(\chi^{2}{ }_{(1)}=106.57, \mathrm{p}<0.001\right)$, and exhibited higher psychological distress as measured with the PHQ- 4 than the OCG (median $_{\mathrm{PSG}}=4, \mathrm{IQR}_{\mathrm{PSG}}=4$, medi$\mathrm{an}_{\mathrm{OCG}}=1, \mathrm{IQR}_{\mathrm{OCG}}=4, \mathrm{U}=4,125, \mathrm{p}<0.001, \eta^{2}=0.20$ ).

Group comparison of SHI total scores indicated a trend (by applying a significance level for $\alpha$ at $\mathrm{p}<0.01$ ) towards less endorsed SHI items in the PSG compared to the OCG $\left(\right.$ median $_{\mathrm{PSG}}=1, \mathrm{IQR}_{\mathrm{PSG}}=2$, median $\left._{\mathrm{OCG}}=1, \mathrm{IQR}_{\mathrm{OCG}}=2.25, \mathrm{U}=7,241, \mathrm{p}=0.033, \eta^{2}=0.02\right)$. At least one type of self-harm behavior during the lifespan was admitted by 72 patients of the PSG and 78 individuals of the OCG (51.8 vs. $\left.63.9 \%, \chi^{2}{ }_{(1)}=3.91, p=0.048\right)$. The results of logistic regressions using Firth's bias reduction method [21] with at least one SHI item endorsed as dependent variable, group as categorical predictor (PSG as baseline), and age or BMI or PHQ-4 as continuous control variable (3 separate models) indicated that only PHQ-4 had a positive effect on the odds ratio. Controlling for PHQ-4, the regression model showed an effect of group on the occurrence of at least one type of self-harm behavior by a factor of 4.30 (95\% CI 2.29-8.42, p < 0.001). For PHQ-4, a one-unit increase in PHQ-4 resulted in an increase of the odds ratio by $38 \%$ (95\% CI 6-55\%, p < 0.001). The comparison between men and women within both groups did not indicate gender differences with respect to SHI total scores or to the prevalence of any lifetime self-harm behavior (results not reported).

The endorsed SHI items by group are presented in table 1 . In both groups, most SHI items were seldom endorsed. There were almost no group differences. Only 'Losing a job on purpose' (item \#17) was more often reported in the OCG than in the PSG. 'Have you ever intentionally, or on purpose attempted suicide' (item \#18) was endorsed by 13 preoperative patients and 15 community controls ( 9.4 vs. $12.4 \%$ for PSG and OCG). Of those persons, 10 preoperative patients and 14 individuals of the OCG provided information on the number of suicide attempts, without significant group differences $\left(\right.$ median $_{P S G}=1.00$, range $_{P S G} 1-5$ vs. median $_{\text {OCG }}=1.00$, range $_{\text {OCG }} 1-4 ; U=65.50, \mathrm{p}=0.796, \eta^{2}=0.003$ ).

\section{Discussion}

Contrary to our prediction, bariatric surgery candidates tended toward less self-harm during the lifespan than community control participants with grade $2 / 3$ obesity. While controlling the analyses for current psychological distress, the odds of any self-harm behavior (vs. no self-harm) were higher in the OCG in comparison to the PSG. One explanation for the unexpected finding could be that some preoperative patients may have withheld symptoms of self-harm or engaged in 'impression management' due to concerns that the admission of self-harm (e.g., substance abuse, making medical situations worse, past suicide attempts) would negatively affect their surgery eligibility or social desirability. However, all patients 
Müller et al.: Lifetime Self-Harm Behaviors Are Not More Prevalent in Bariatric Surgery Candidates than in Community Controls with Obesity

Table 1. Lifetime self-harm behaviors in the pre-surgery group (PSG, $n=139$ ) and the obese control group (OCG, $\mathrm{n}=122$ ) as measured with the Self-Harm Inventory [1]

\begin{tabular}{|c|c|c|c|c|}
\hline \multirow[t]{2}{*}{ Self-harm behaviors } & \multirow[t]{2}{*}{ PSG, n (\%) } & \multirow[t]{2}{*}{ OCG, n (\%) } & \multicolumn{2}{|c|}{ PSG vs. OCG } \\
\hline & & & $\chi^{2}(1)$ & $\mathrm{p}^{\mathrm{a}}$ \\
\hline Overdosed & $6(4.4)$ & $8(6.6)$ & 0.56 & 0.584 \\
\hline Cut yourself & $11(7.9)$ & $11(9.0)$ & 0.10 & 0.825 \\
\hline Burned yourself & $1(0.7)$ & $3(2.5)$ & 1.30 & 0.343 \\
\hline Hit yourself & $3(2.2)$ & $4(3.3)$ & 0.30 & 0.709 \\
\hline Banged your head & $3(2.2)$ & $5(4.1)$ & 0.83 & 0.479 \\
\hline Abused alcohol & $11(7.9)$ & $21(17.4)$ & 5.34 & 0.024 \\
\hline Driven recklessly & $5(3.6)$ & $10(8.2)$ & 2.54 & 0.181 \\
\hline Scratched yourself & $15(10.8)$ & $15(12.4)$ & 0.16 & 0.702 \\
\hline Prevented wounds from healing & $8(5.8)$ & $10(8.2)$ & 0.60 & 0.472 \\
\hline Made medical situations worse & $2(1.4)$ & $10(8.3)$ & 6.78 & 0.015 \\
\hline Been promiscuous & $8(5.8)$ & $6(4.9)$ & 0.09 & 0.791 \\
\hline Set yourself up in a relationship to be rejected & $2(1.4)$ & $6(4.9)$ & 2.61 & 0.152 \\
\hline Abused prescription medication & $1(0.7)$ & $7(5.7)$ & 5.46 & 0.028 \\
\hline Distanced yourself from God as punishment & $1(0.7)$ & $2(1.7)$ & 0.48 & 0.600 \\
\hline Engaged in emotionally abusive relationships & $15(10.9)$ & $7(5.8)$ & 2.14 & 0.182 \\
\hline Engaged in sexually abusive relationships & $5(3.6)$ & $9(7.4)$ & 1.80 & 0.271 \\
\hline Lost a job on purpose & $21(15.1)$ & $46(38.0)$ & 17.75 & $<0.001$ \\
\hline Attempted suicide & $13(9.4)$ & $15(12.4)$ & 0.62 & 0.548 \\
\hline Exercised an injury & $8(5.8)$ & $10(8.3)$ & 0.61 & 0.471 \\
\hline Tortured yourself with self-defeating thoughts & $55(40.1)$ & $47(38.8)$ & 0.05 & 0.899 \\
\hline Starved yourself to hurt yourself & $6(4.3)$ & $3(2.5)$ & 0.69 & 0.508 \\
\hline Abused laxatives to hurt yourself & 0 & $3(2.5)$ & - & - \\
\hline
\end{tabular}

${ }^{\text {a}}$ Exact Fisher's test, two-tailed.

have been assured that the current research data were collected independently of the surgery approval process and clinical care. An alternative explanation could be that, although preoperative patients tended to less self-harm behaviors in general, they could have applied specific self-harm behaviors more frequently than community control participants. At least with regard to suicide attempts, however, the number of reported suicide attempts did not differ between the two groups.

The group difference in endorsement rates of the SHI item 'Have you ever lost a job on purpose' (higher rates in the OCG) is difficult to explain. It is possible that some participants misunderstood this item and gave an affirmative answer because they lost a job to escape from negative job-related experiences and not to intentionally hurt themselves. The high prevalence of 'torturing with self-defeating thoughts' (item \#20) in both groups ( $40.1 \%$ and $38.8 \%$ for PSG and OCG, respectively) indicates a high level of depressive symptoms in people with obesity, which is in accordance with the literature concerning the link between obesity and depression [22]. In a similar vein, our results suggest high rates of suicide attempts in both groups ( $9.4 \%$ and $12.4 \%$ for PSG and OCG, respectively) not differing from each other, and exceeding lifetime estimates in the population (e.g., $2.7 \%$ according to the World Mental Health Initiative) [23]. This finding echoes past results suggesting an elevated suicide risk in persons with extreme obesity [24-26]. Furthermore, the proportion of patients with past suicide attempts in the current preoperative sample (9.4\%) was very close to that from an earlier preoperative group (9.1\%) investigated by Sansone et al. [12].

The major strength of the current study is that it provides information on a broad range of self-harm behaviors among bariatric surgery candidates in comparison to community 
control participants with BMI $35+\mathrm{kg} / \mathrm{m}^{2}$. Nevertheless, there are several shortcomings that are to be considered when interpreting the results. The groups were not matched, resulting in group differences (i.e., BMI, age) that might have biased the outcome. However, the results of logistic regressions suggest that, apart from the effect of group, only PHQ-4 had a positive effect on the odds of any self-harm behavior (vs. no self-harm), while age and BMI did not influence the result. Future case control studies should investigate self-harm in bariatric surgery patients and matched non-operated patients with obesity to eliminate confounding. Furthermore, the present data are cross-sectional and cannot be used to establish causality between self-harm and other variables. Self-harm behaviors were assessed during the lifespan, whereas weight and height referred to the time of assessment. Past changes in BMI or weight cycling could not be taken into account. The BMI data are limited by self-reports of weight and height and may therefore correlate more with perceived than with actual BMIs. However, studies in candidates seeking gastric bypass surgery [27] or patients following bariatric surgery $[28,29]$ have revealed minimal discrepancies between reported and measured weight and height. In addition, all data are based on self-ratings and may not reflect true prevalence rates of self-harm. Last but not least, a selection bias has to be considered given that $41 \%$ of potential candidates for the study had rejected participation.

\section{Conclusion}

Overall, the findings of the present study do not support the assumption that bariatric surgery candidates suffer from more lifetime self-harm than individuals with class $2 / 3$ obesity from the community. Further studies are needed to investigate self-harm in bariatric surgery patients prior to and following surgery compared to non-operated patients with obesity.

\section{Ethical Standards}

The authors assert that all procedures contributing to this work comply with the Helsinki Declaration of 1975, as revised in 2008, and have been approved by an independent Ethics Committee at the Hannover Medical School. Informed consent was obtained from all bariatric surgery candidates.

\section{Funding}

This research did not receive any specific grant from funding agencies in the public, commercial, or notfor-profit sectors.

\section{Disclosure Statement}

The authors declare no conflict of interest. 
Müller et al.: Lifetime Self-Harm Behaviors Are Not More Prevalent in Bariatric Surgery Candidates than in Community Controls with Obesity

\section{References}

1 Sansone RA, Wiederman MW, Sansone LA: The self-harm inventory (SHI): development of a scale for identifying self-destructive behaviors and borderline personality disorder. J Clin Psychol 1998;54:973-983.

2 Claes L, Vandereycken W: Self-injurious behavior: differential diagnosis and functional differentiation. Compr Psychiatry 2007;48:137-144.

3 Backman 0, Stockeld D, Rasmussen F, Naslund E, Marsk R: Alcohol and substance abuse, depression and suicide attempts after Roux-en-Y gastric bypass surgery. Br J Surg 2016;103:1336-1342.

-4 Bhatti JA, Nathens AB, Thiruchelvam D, Grantcharov T, Goldstein BI, Redelmeier DA: Self-harm emergencies after bariatric surgery: a population-based cohort study. JAMA Surg 2016;151:226-232.

-5 Kovacs Z, Valentin JB, Nielsen RE: Risk of psychiatric disorders, self-harm behaviour and service use associated with bariatric surgery. Acta Psychiatr Scand 2017;135:149-158.

-6 Tindle HA, Omalu B, Courcoulas A, Marcus M, Hammers J, Kuller LH: Risk of suicide after long-term follow-up from bariatric surgery. Am J Med 2010;123:1036-1042.

7 Mitchell JE, Crosby R, de Zwaan M, Engel S, Roerig J, Steffen K, Gordon KH, Karr T, Lavender J, Wonderlich S: Possible risk factors for increased suicide following bariatric surgery. Obesity (Silver Spring) 2013;21:665672.

-8 Peterhänsel C, Petroff D, Klinitzke G, Kersting A, Wagner B: Risk of completed suicide after bariatric surgery: a systematic review. Obes Rev 2013;14:369-382.

-9 Lagerros YT, Brandt L, Hedberg J, Sundbom M, Boden R: Suicide, self-harm, and depression after gastric bypass surgery: a nationwide cohort study. Ann Surg 2017;265:235-243.

10 Gribsholt SB, Thomsen RW, Svensson E, Richelsen B: Overall and cause-specific mortality after Roux-en-Y gastric bypass surgery: a nationwide cohort study. Surg Obes Relat Dis 2017;13:581-587.

11 Gribsholt SB, Svensson E, Richelsen B, Raundahl U, Sorensen HT, Thomsen RW: Rate of acute hospital admissions before and after Roux-en-Y gastric bypass surgery: a population-based cohort study. Ann Surg 2018; 267:319-325.

12 Sansone RA, Wiederman MW, Schumacher DF, Routsong-Weichers L: The prevalence of self-harm behaviors among a sample of gastric surgery candidates. J Psychosom Res 2008;65:441-444.

13 Müller A, Claes L, Smits D, Brähler E, de Zwaan M: Prevalence and correlates of self-harm in the German general population. PLoS One 2016;11:e0157928.

14 Duarte-Guerra LS, Coelho BM, Santo MA, Wang YP: Psychiatric disorders among obese patients seeking bariatric surgery: results of structured clinical interviews. Obes Surg 2015;25:830-837.

15 Dawes AJ, Maggard-Gibbons M, Maher AR, Booth MJ, Miake-Lye I, Beroes JM, Shekelle PG: Mental health conditions among patients seeking and undergoing bariatric surgery: a meta-analysis. JAMA 2016;315:150-163.

16 Mitchell JE, Selzer F, Kalarchian MA, Devlin MJ, Strain GW, Elder KA, Marcus MD, Wonderlich S, Christian NJ, Yanovski SZ: Psychopathology before surgery in the longitudinal assessment of bariatric surgery-3 (LABS-3) psychosocial study. Surg Obes Relat Dis 2012;8:533-541.

$\checkmark 17$ Malik S, Mitchell JE, Engel S, Crosby R, Wonderlich S: Psychopathology in bariatric surgery candidates: a review of studies using structured diagnostic interviews. Compr Psychiatry 2014;55:248-259.

18 Bhatti JA, Nathens AB, Redelmeier DA: Deliberate self-harm following bariatric surgery-reply. JAMA Surg 2016;151:585-586.

19 Kroenke K, Spitzer RL, Williams JB, Löwe B: An ultra-brief screening scale for anxiety and depression: The PHQ-4. Psychosomatics 2009;50:613-621.

20 Cohen J: Statistical Power Analysis for the Behavioral Sciences. Hillsdale, Lawrence Erlbaum Associates, 1988.

21 Firth D: Bias reduction of maximum likelihood estimates. Biometrika 1993;80:27-38.

22 de Wit L, Luppino F, van Straten A, Penninx B, Zitman F, Cuijpers P: Depression and obesity: a meta-analysis of community-based studies. Psychiatry Res 2010;178:230-235.

23 Nock MK, Borges G, Bromet EJ, Alonso J, Angermeyer M, Beautrais A, Bruffaerts R, Chiu WT, de Girolamo G, Gluzman S, de Graaf R, Gureje O, Haro JM, Huang Y, Karam E, Kessler RC, Lepine JP, Levinson D, Medina-Mora ME, Ono Y, Posada-Villa J, Williams D: Cross-national prevalence and risk factors for suicidal ideation, plans and attempts. Br J Psychiatry 2008;192:98-105.

24 Wagner B, Klinitzke G, Brähler E, Kersting A: Extreme obesity is associated with suicidal behavior and suicide attempts in adults: results of a population-based representative sample. Depress Anxiety 2013;30:975-981.

25 Elovainio M, Shipley MJ, Ferrie JE, Gimeno D, Vahtera J, Marmot MG, Kivimaki M: Obesity, unexplained weight loss and suicide: the original Whitehall study. J Affect Disord 2009;116:218-221.

-26 Dong C, Li WD, Li D, Price RA: Extreme obesity is associated with attempted suicides: results from a family study. Int J Obes (Lond) 2006;30:388-390.

27 White MA, Masheb RM, Burke-Martindale C, Rothschild B, Grilo CM: Accuracy of self-reported weight among bariatric surgery candidates: the influence of race and weight cycling. Obesity (Silver Spring) 2007;15:27612768.

28 Ivezaj V, Grilo CM: Accuracy of self-reported height and weight among sleeve gastrectomy patients with disordered eating. Obes Surg 2017;27:267-270.

29 Christian NJ, King WC, Yanovski SZ, Courcoulas AP, Belle SH: Validity of self-reported weights following bariatric surgery. JAMA 2013;310:2454-2456. 